\title{
Randomised controlled trial of adjunctive inspiratory muscle training for patients with COPD
}

\author{
Noppawan Charususin, 1,2,3 Rik Gosselink, ${ }^{1,2}$ Marc Decramer, ${ }^{1}$ Heleen Demeyer, ${ }^{1,2}$ \\ Alison McConnell, ${ }^{4}$ Didier Saey, ${ }^{5}$ François Maltais, ${ }^{5}$ Eric Derom, ${ }^{6}$ Stefanie Vermeersch, ${ }^{6}$ \\ Yvonne F Heijdra, ${ }^{7}$ Hanneke van Helvoort, ${ }^{7}$ Linda Garms, ${ }^{7}$ Tessa Schneeberger, ${ }^{8}$ \\ Klaus Kenn, ${ }^{8,9}$ Rainer Gloeckl, ${ }^{8,10}$ Daniel Langer ${ }^{1,2}$
}

\begin{abstract}
- Additional material is published online only. To view please visit the journal online (http://dx.doi.org/10.1136/ thoraxjnl-2017-211417).
\end{abstract}

For numbered affiliations see end of article.

\section{Correspondence to}

Dr Daniel Langer, Department of Rehabilitation Sciences, KU Leuven, Leuven 3001, Belgium daniel.langer@kuleuven.be

Received 14 December 2017 Revised 16 May 2018

Accepted 28 May 2018 Published Online First 18 June 2018

\section{Linked}

- http://dx.doi.org/10.1136/ thoraxjnl-2018-212070

Check for updates

To cite: Charususin $\mathrm{N}$, Gosselink R, Decramer M, et al. Thorax

2018:73:942-950.

\begin{abstract}
Background This study aimed to investigate whether adjunctive inspiratory muscle training (IMT) can enhance the well-established benefits of pulmonary rehabilitation (PR) in patients with COPD.
\end{abstract}

Methods 219 patients with COPD (FEV : 42\% $16 \%$ predicted) with inspiratory muscle weakness (PImax: $51 \pm 15 \mathrm{~cm} \mathrm{H}$ O) were randomised into an intervention group (IMT+PR; $n=110$ ) or a control group (ShamIMT+PR; $n=109$ ) in this double-blind, multicentre randomised controlled trial between February 2012 and October 2016 (ClinicalTrials.gov NCT01397396). Improvement in 6 min walking distance (6MWD) was a priori defined as the primary outcome. Prespecified secondary outcomes included respiratory muscle function and endurance cycling time.

Findings No significant differences between the intervention group $(n=89)$ and the control group $(n=85)$ in improvements in 6MWD were observed $(0.3 \mathrm{~m}$, $95 \% \mathrm{Cl}-13$ to $14, \mathrm{p}=0.967)$. Patients who completed assessments in the intervention group achieved larger gains in inspiratory muscle strength (effect size: 1.07, $p<0.001$ ) and endurance (effect size: $0.79, p<0.001$ ) than patients in the control group. $75 \mathrm{~s}$ additional improvement in endurance cycling time $(95 \% \mathrm{Cl} 1$ to $149, p=0.048$ ) and significant reductions in Borg dyspnoea score at isotime during the cycling test $(95 \% \mathrm{Cl}-1.5$ to $-0.01, \mathrm{p}=0.049)$ were observed in the intervention group.

Interpretation Improvements in respiratory muscle function after adjunctive IMT did not translate into additional improvements in 6MWD (primary outcome). Additional gains in endurance time and reductions in symptoms of dyspnoea were observed during an endurance cycling test (secondary outcome)

Trial registration number NCT01397396; Results.

\section{INTRODUCTION}

COPD is a major cause of chronic morbidity and mortality worldwide. ${ }^{1}$ Respiratory muscle dysfunction, which is frequently observed in patients with COPD ${ }^{2}$ plays a key role in the perception of dyspnoea $^{34}$ and contributes to exercise intolerance in these patients. ${ }^{56}$

Pulmonary rehabilitation (PR) is standard care for patients with COPD and results in statistically significant and clinically relevant improvements in exercise capacity and health-related quality of life. ${ }^{78}$ Inspiratory muscle training (IMT) has been

\section{Key messages}

What is the key question?

- Can adjunctive inspiratory muscle training enhance the well-established benefits of pulmonary rehabilitation in selected COPD patients with inspiratory muscle weakness?

What is the bottom line?

- Improvements in respiratory muscle function beyond those induced by general exercise training did not result in additional improvements in 6 min walking distance (primary outcome).

Why read on?

- The observed between-group difference in endurance cycling time could serve as a basis for sample size calculations using endurance tests as primary outcomes in future studies of adjunct interventions.

extensively studied in the last decades in patients with COPD. ${ }^{9}$ However, due to differing interpretations of the existing evidence, in combination with limited resources and time constraints during PR programmes, this adjunctive therapy is at this moment not included in about $50 \%$ of these programmes. $^{9-14}$ IMT as a stand-alone therapy improves inspiratory muscle function (strength and endurance), decreases symptoms of dyspnoea and improves exercise capacity. ${ }^{9}$ The value of adjunctive IMT during PR is less clear. While adjunctive IMT in combination with general exercise training (GET) resulted in significant additional improvements in respiratory muscle function beyond the effects of GET alone, its additional effects on outcomes such as exercise capacity and quality of life are insufficiently supported by data so far. ${ }^{79}$ Based on subgroup analyses in meta-analyses and general physiological considerations, it has been recommended that future studies of adjunctive IMT in patients with COPD should focus specifically on patients with exercise-induced dyspnoea and inspiratory muscle weakness. ${ }^{7915}$

The present study examined the effects of adding a largely unsupervised, but well-controlled adjunctive IMT programme in the context of an adequately powered, multicentre, double-blind, randomised 
controlled trial (RCT) design, in selected COPD patients with inspiratory muscle weakness. We wanted to answer the question whether IMT can be a useful adjunctive therapy during PR for patients with COPD. We hypothesised that adjunctive IMT would result in additional improvements in functional exercise capacity (primary outcome) and inspiratory muscle function compared with rehabilitation alone.

\section{METHODS}

This study was registered in an international trial registry database (ClinicalTrials.gov: NCT01397396). Clinically stable COPD patients with reduced maximal inspiratory mouth pressure (PImax $<60 \mathrm{~cm} \mathrm{H}_{2} \mathrm{O}$ or $<50 \%$ of the predicted normal value) participated in the study between February 2012 and October 2016. ${ }^{9}$

More information about study procedures can be found in an extended 'Materials and methods' section and in a video tutorial (see online supplementary video files 1,2,3, and 4), as well as in a previously published study protocol. ${ }^{16}$

\section{Interventions}

Both groups performed an identical GET programme. The training volume ranged from 20 sessions (Germany) to 36 sessions (other centres). Training frequency ranged from 3 to 5 sessions per week. Duration of training sessions was around $60 \mathrm{~min}$. Patients performed endurance training or interval training at moderate to high intensities. Training intensities during GET were progressively increased during the course of the programme based on symptom scores. Training intensities corresponded to Borg CR-10 scale ratings of 4-6 on dyspnoea sensation. ${ }^{16}$ Physiotherapists providing this intervention were blinded to group allocation of patients. All participants (intervention and control group) were led to believe that they followed an active adjunctive IMT intervention during the GET intervention. This was done in order to improve adherence with the intervention in the control group and to ensure placebo treatment effects in both groups. ${ }^{17}$ IMT in both groups was performed daily using the PowerBreathe KHP2 device (POWERbreatheKHP2, HaB International, Southam, UK) according to previously described methods. ${ }^{18}$ The training intensity (average external load $\left(\mathrm{cm} \mathrm{H}_{2} \mathrm{O}\right)$ ) and training quality (average mean power per breath and total work of breathing during one session of 30 breaths) were registered and stored during all training sessions. During weekly supervised sessions (7\% of all training sessions), data on training quality during the unsupervised sessions were reviewed and patients received instructions and feedback on how to optimise their home training efforts. Training intensity in the intervention group was set initially at a load of approximately $50 \%$ of patients' maximal inspiratory mouth pressure (PImax). This initial load was then continuously and gradually increased to the highest tolerable intensity during each of the supervised sessions. ${ }^{18}$ Training intensity in the control group was set at $10 \%$ baseline PImax and was not modified throughout the intervention period.

\section{Outcome measurements}

The primary outcome $6 \mathrm{~min}$ walking distance $(6 \mathrm{MWD})$ was assessed according to a standardised published protocol and related to reference values. ${ }^{19}$ Secondary outcomes included respiratory muscle function (strength and endurance), maximal and endurance cycling exercise capacity, pulmonary function, limb muscle forces and objectively measured physical activity
(DynaPort Minimod, McRoberts, The Hague, The Netherlands). All outcome assessors were blinded to group allocation.

\section{Sample size calculation}

To detect a minimally clinically important difference (MCID) between groups of $26 \mathrm{~m}$ in the $6 \mathrm{MWD},{ }^{20}$ assuming an $\mathrm{SD}$ of the within-group differences in the $6 \mathrm{MWD}$ at the end of the intervention period of $60 \mathrm{~m}$ in both groups with a degree of certainty (statistical power) of $80 \%$ and a risk for a type I error $(\alpha)<5 \%$, minimally 85 patients were calculated to be included in each group, given an anticipated dropout rate of $30 \%$.

\section{Statistical analysis}

All data were analysed using a modified intention-to-treat approach (SPSS V.24 (IBM) and SAS V.9.4 (SAS Institute)). This means that we did not consider patients who had missing outcome data due to loss to follow-up in the analysis. Consequently, no imputation for missing data was performed and a so-called 'complete/available case analysis' was performed. ${ }^{21}$ The missing data were interpreted as 'missing at random'. Differences between groups after the intervention were compared, adjusting for values of the respective outcomes at baseline, using analysis of covariance. ${ }^{22}$ Because randomisation was performed by centre, the adjustment by centre was tested. Additional exploratory analyses were performed to study (1) the impact of IMT characteristics on improvements in PImax and (2) the relationship between improvements in PImax and GET outcomes. More details on analyses by 'centre' and exploratory analyses are provided in the online data supplement. To account for the potential impact of differences in training volume between GET programme offered in the different centres on outcomes, we also tested the effect of 'centre' on treatment effects using centre*intervention effect. In post-hoc tests, we subsequently compared the effects in the centre offering 20 sessions GET intervention with the other centres offering 36 sessions.

\section{RESULTS}

Between February 2012 and October 2016, 998 patients who entered the rehabilitation programme in the different centres were screened for study participation. A diagram summarising the flow of participants through the study is presented in figure 1. The most frequent reason for non-eligibility was not meeting the PImax inclusion criteria (73\% of excluded subjects). At the end of the study, data were available for the primary outcome in $85 \%$ of subjects from the intervention group and $83 \%$ of participants in the control group. Baseline characteristics of included patients were not significantly different between the two groups (table 1). Comparisons between completers and patients who were lost to follow-up are summarised in the online supplementary table E1.

\section{Changes in exercise capacity and GET progression}

Results of different tests for exercise related outcomes are presented in table 2 . No statistically significant between-group differences in improvements in 6MWD (primary outcome) were observed. Endurance cycling time (secondary outcome) improvement was significantly greater in the intervention group with significant reductions in dyspnoea symptoms at isotime during the test (table 2). Both cycling and treadmill training intensities during GET sessions were slightly higher in the intervention group without reaching statistical significance $(p=0.212$ and 0.657 for cycling and treadmill walking, respectively; see online supplementary figure E1). 


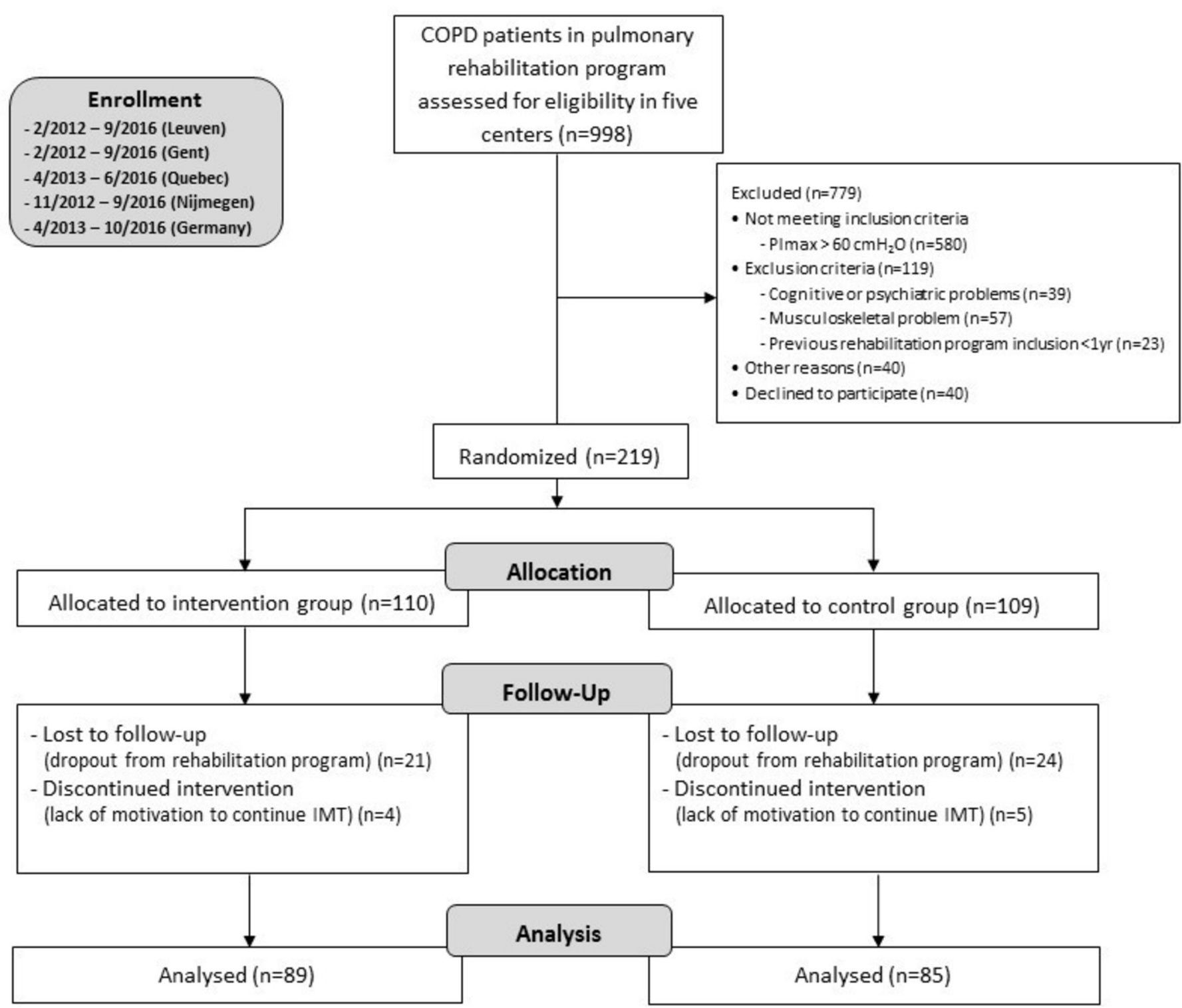

Figure 1 A diagram summarising the flow of participants through the study.

\section{IMT progression}

The intervention group completed $79 \% \pm 4 \%$ of prescribed IMT sessions while the control group completed $81 \% \pm 4 \%$ of prescribed sessions. The intervention group increased their training load from $47 \% \pm 2 \%$ of their baseline PImax in the first week of training to $84 \% \pm 4 \%$ of their baseline PImax in week 12 (see online supplementary figure E2). Data on the weekly sum of total work performed during all IMT sessions (based on pressure and volume data recorded and stored by the training devices) are presented in figure 2 . The average Borg CR-10 scale inspiratory effort scores for breathing during the final minute of the supervised IMT sessions were $3.5 \pm 0.3$ and $2.6 \pm 0.3$ for intervention and control group, respectively.

\section{Changes in respiratory muscle function}

Patients in the intervention group exhibited significantly larger improvements in inspiratory, but not expiratory muscle strength compared with the control group (table 3). During the endurance breathing test, the magnitude of improvement in time to the limit of tolerance, average mean power per breath and total work were also significantly larger in the intervention group (table 3).

Changes in pulmonary function, quality of life and other secondary outcomes

The intervention group exhibited a significantly larger increase in FVC, both in comparison to baseline and to the control group $(\mathrm{p}<0.001$ and $\mathrm{p}=0.028$, respectively). There were no statistically significant changes in other pulmonary function variables between the two groups (online supplementary table E2). While both groups exhibited statistically significant and clinically relevant improvements within groups, there were no statistically significant differences between groups in all domains of the CRQ (online supplementary table E3). Quadriceps and handgrip strength were also both significantly improved within both groups after training with no statistically significant betweengroup differences. There was no statistically significant difference in the change in the number of steps per day within or between the two groups after the intervention. 
Table 1 Baseline characteristics of patients

\begin{tabular}{|c|c|c|}
\hline Variables & $\begin{array}{l}\text { Intervention group } \\
(\mathrm{n}=110)\end{array}$ & $\begin{array}{l}\text { Control group } \\
(n=109)\end{array}$ \\
\hline Age (years) & $66 \pm 8$ & $65 \pm 7$ \\
\hline Gender (F/M) & $58 / 52$ & $66 / 43$ \\
\hline BMI $\left(\mathrm{kg} / \mathrm{m}^{2}\right)$ & $25 \pm 6$ & $24 \pm 6$ \\
\hline $\mathrm{FEV}_{1}$ (\%predicted) & $40 \pm 15$ & $43 \pm 17$ \\
\hline FVC (\%predicted) & $70 \pm 19$ & $77 \pm 23$ \\
\hline $\mathrm{FEV}_{1} / \mathrm{FVC}(\%)$ & $45 \pm 14$ & $45 \pm 13$ \\
\hline TLC (\%predicted) & $123 \pm 25$ & $124 \pm 24$ \\
\hline FRC (\%predicted) & $180 \pm 47$ & $179 \pm 44$ \\
\hline RV (\%predicted) & $213 \pm 64$ & $206 \pm 70$ \\
\hline DLCO (\%predicted) & $42 \pm 19$ & $41 \pm 21$ \\
\hline PImax $\left(\mathrm{cm} \mathrm{H}_{2} \mathrm{O}\right)$ & $52 \pm 14$ & $51 \pm 12$ \\
\hline PImax (\%predicted) & $51 \pm 15$ & $52 \pm 14$ \\
\hline Endurance breathing time (s) & $243 \pm 109$ & $242 \pm 93$ \\
\hline Inspiratory load (\%PImax) & $56 \pm 12$ & $56 \pm 15$ \\
\hline Peak VO ${ }_{2}$ (\%predicted) & $66 \pm 28$ & $63 \pm 26$ \\
\hline Peak work rate (\%predicted) & $45 \pm 25$ & $45 \pm 22$ \\
\hline Endurance cycle time (s) & $262 \pm 124$ & $287 \pm 159$ \\
\hline $6 \mathrm{MWD}(\mathrm{m})$ & $346 \pm 118$ & $355 \pm 104$ \\
\hline 6MWD (\%predicted) & $56 \pm 20$ & $57 \pm 18$ \\
\hline Quadriceps strength (\%predicted) & $70 \pm 23$ & $81 \pm 36$ \\
\hline Hand grip strength (\%predicted) & $87 \pm 24$ & $88 \pm 25$ \\
\hline PA (steps/day) & $3679 \pm 2234$ & $4238 \pm 1853$ \\
\hline CRQ dyspnoea & $15 \pm 6$ & $15 \pm 5$ \\
\hline mMRC & $2.5 \pm 1.2$ & $2.6 \pm 1.0$ \\
\hline
\end{tabular}

Data are presented as mean \pm SD.

6MWD, 6 min walking distance; BMI, body mass index; CRQ,

chronic respiratory questionnaire; $\mathrm{DLCO}$, diffusing capacity of the lungs for carbon monoxide; FRC, functional residual capacity; $\mathrm{MMRC}$, modified Medical Research Council dyspnoea scale; $\mathrm{PA}$, physical activity; peak $\mathrm{VO}_{2}$, peak oxygen uptake; peak work rate, peak power output during a maximal incremental cycle ergometry test; PImax, maximal inspiratory mouth pressure; \%predicted, percentage of the predicted normal value; RV, residual volume; TLC, total lung capacity.

\section{Impact of centre on outcomes}

Interactions between centres and between-group post-treatment differences in PImax $(p=0.007)$, progression of training intensity during GET $(p=0.200)$, endurance cycling time $(p=0.007)$ and 6MWD $(\mathrm{p}=0.040)$ were observed. One of the centres offering a 36 session programme (32\% of total inclusions) consistently exceeded between group differences in the centre offering 20 sessions (36\% of total inclusions). In the other centres offering 36 sessions (combined 32\% of total inclusions), between-group differences in these outcomes were consistently smaller than in the centre offering a lower training volume (see online supplementary table E4).

\section{Correlates of improvements in inspiratory muscle strength}

Both training quality indices registered during IMT sessions (ie, total inspiratory work performed per session and average peak power per session) and progression in training intensity were significantly related to the magnitude of improvements achieved in PImax (figure 3A-C). Conversely, average training volume (the number of sessions stored by the TFRL devices) was not significantly associated with changes in PImax ( $p$-fortrend $=0.129$ ). In a stepwise multiple regression analyses, total work performed during IMT sessions (partial $\left.\mathrm{r}^{2}: 0.24\right)$, baseline PImax (partial $r^{2}: 0.08$ ) and training compliance (partial $r^{2}: 0.01$ ) were significantly related to the improvements in PImax after training $\left(\mathrm{F}(3,139)=22.9, \mathrm{p}<0.001, \mathrm{R}^{2}=0.33\right)$. More detailed results of the regression analyses are provided in online supplementary table E5. Subsequently, improvements in PImax were related to the progression in cycling training intensity during the GET programme, as well as to increases in 6MWD and endurance cycling time after the training period (figure $4 \mathrm{~A}-\mathrm{C}$ ).

\section{DISCUSSION}

This study is the first adequately powered RCT that investigated the effects of adjunctive IMT on outcomes beyond respiratory muscle function in patients with COPD who had been selected based on the presence of inspiratory muscle weakness. There was no statistically significant difference between groups for improvements in 6MWD (primary outcome). Patients in the intervention group however achieved larger gains in respiratory muscle function and also endurance exercise capacity (prespecified secondary outcome) increased significantly more in the intervention group. Significant reductions in dyspnoea symptoms at isotime during the cycling test were also observed in favour of the intervention group.

Contrary to our initial hypothesis, larger gains in respiratory muscle function in the intervention group did not translate into further improvements in 6MWD compared with the control group. Changes in the intervention group and the control group were both equally large and fall within the range of a MCID of $25-35 \mathrm{~m} .{ }^{23}$ These findings are in line with results from a recent systematic review of RCT's studying adjunctive interventions during PR. In this meta-analysis, none of the studied adjunctive interventions (eg, leg muscle strength training, IMT, non-invasive ventilation or nutritional supplementation) were able to induce statistically significant additional improvements in $6 \mathrm{MWD} .^{24}$ In contrast to the results of the $6 \mathrm{MWD}$, significantly larger improvements in cycling endurance time and significantly greater reductions in dyspnoea symptoms at isotime were observed in the intervention group in the current study. The average additional improvement in endurance cycling time of $75 \mathrm{~s}$ falls within a range of 46-105 s that has recently been defined as an MCID for constant-load cycle endurance time improvements in response to pharmacological interventions. ${ }^{25}$ The superior responsiveness of endurance exercise tests compared with the 6MWD test is in line with several other recent reports comparing responses in these outcome measures after both pharmacological and non-pharmacological interventions in patients with COPD. ${ }^{26-28}$ These differences are probably related to the different characteristics of physiological responses elicited by the two tests. ${ }^{26} 29$ In hindsight we believe that our expected between-group difference in $6 \mathrm{MWD}$ of $26 \mathrm{~m}$ did not represent a realistic magnitude of between-group differences in 6MWD in studies of adjunct interventions to PR. Our data rather indicate that a more sensitive outcome (ie, endurance walking or cycling test) might be more useful as a primary outcome. In the absence of a clinically meaningful difference for adjunct interventions, the magnitude of our observed between-group differences both in endurance cycling time and symptoms at isotime could serve as a basis for sample size calculations using endurance tests as primary outcomes in future studies of adjunct interventions. It is unlikely that the type of analysis (intention to treat based on 'complete/ availablecase analysis') had a major impact on the results since the 'completers' of the study did not differ significantly from 'non-completers' with regard to baseline characteristics that 
Table 2 Changes in exercise-related outcomes

\begin{tabular}{|c|c|c|c|c|c|c|}
\hline \multirow[b]{2}{*}{ Variables } & \multicolumn{2}{|c|}{ Intervention group } & \multicolumn{2}{|c|}{ Control group } & \multirow{2}{*}{$\begin{array}{l}\text { Adjusted difference }(95 \% \mathrm{Cl}) \\
\text { at post training }\end{array}$} & \multirow[b]{2}{*}{$P$ values* } \\
\hline & Pre training & Post training & Pre training & Post training & & \\
\hline \multicolumn{7}{|l|}{ Functional exercise capacity } \\
\hline $6 \mathrm{MWD}(\mathrm{m})$ & $353(116)$ & $388(113) \dagger$ & $374(102)$ & $407(105) \dagger$ & 0.3 (-13 to 14$)$ & 0.967 \\
\hline Dyspnoea post 6MWD & $5.4(2.2)$ & $5.7(2.3)$ & $5.5(2.2)$ & $5.4(2.1)$ & $0.2(-0.3$ to 0.8$)$ & 0.400 \\
\hline Leg effort post 6MWD & $4.2(2.3)$ & $4.3(2.0)$ & $4.3(2.4)$ & $4.4(2.0)$ & $-0.1(-0.7$ to 0.4$)$ & 0.630 \\
\hline \multicolumn{7}{|l|}{ Maximal exercise capacity } \\
\hline Peak work rate $(W)$ & $54(21)$ & $64(26) \dagger$ & $54(20)$ & $59(22) \dagger$ & $5.2(-0.4$ to 10.8$)$ & 0.069 \\
\hline Peak VO ${ }_{2}(\mathrm{~mL} / \mathrm{min})$ & $1009(310)$ & $1048(313)$ & 909 (275) & $966(323)$ & $0.01(-0.09$ to 0.11$)$ & 0.881 \\
\hline Peak $V_{E}(L / m i n)$ & $36(11)$ & $37(11)$ & $38(12)$ & $39(15)$ & $-0.5(-3.4$ to 2.3$)$ & 0.703 \\
\hline Dyspnoea post CPET & $6.7(2.6)$ & $6.3(2.7)$ & $5.9(2.3)$ & $6.4(2.1)$ & $-0.5(-1.4$ to 0.5$)$ & 0.324 \\
\hline Leg effort post CPET & $5.8(2.9)$ & $6.0(2.4)$ & $5.9(2.2)$ & $6.1(2.1)$ & $-0.1(-1.0$ to 0.8$)$ & 0.836 \\
\hline \multicolumn{7}{|l|}{ Endurance exercise capacity } \\
\hline Work rate (W) & $42(16)$ & & $44(18)$ & & & \\
\hline Endurance cycle time (s) & $271(126)$ & $496(309) \dagger$ & $303(163)$ & $466(292) \dagger$ & 75 (1 to 149$)$ & 0.048 \\
\hline Dyspnoea post cycle test & $6.1(2.2)$ & $6.0(2.1)$ & $6.1(2.2)$ & $5.9(2.3)$ & $-0.3(-1.0$ to 0.4$)$ & 0.405 \\
\hline Leg effort post cycle test & $6.0(1.9)$ & $5.2(2.1) \dagger$ & $5.5(2.3)$ & $5.5(2.3)$ & $-0.4(-1.2$ to 0.3$)$ & 0.216 \\
\hline Dyspnoea score at isotime & $6.1(2.2)$ & $3.7(1.3) \dagger$ & $5.9(2.0)$ & $4.4(1.9) \dagger$ & $-0.7(-1.5$ to -0.01$)$ & 0.049 \\
\hline Leg effort score at isotime & $6.1(1.9)$ & $4.2(1.7) \dagger$ & $4.9(2.3)$ & $4.5(2.2)$ & $-0.9(-1.7$ to 0.01$)$ & 0.052 \\
\hline
\end{tabular}

Data are presented as mean (SD) and mean $(95 \% \mathrm{Cl})$. Dyspnoea and leg effort scores were evaluated with the modified CR-10 Borg scale. Analyses are based on 169 (6MWD, $97 \%$ of completers), 92 (peak work rate, $53 \%$ of completers) and 139 (endurance cycle time, $80 \%$ of completers) patients.

${ }^{*} \mathrm{P}$ values are reported for between-group comparisons (analysis of covariance of post-training values adjusted for baseline values as covariates).

†A statistically significant difference within groups $(p<0.05)$,

6MWD, 6 min walking distance; $C P E T$, cardiopulmonary exercise test; peak VE, peak pulmonary ventilation; peak $\mathrm{VO}_{2^{\prime}}$ peak oxygen uptake; peak work rate, peak power output.

were identified as confounding variables (see online supplementary tables E1 and E6).

In the current study, we found $23 \mathrm{~cm} \mathrm{H}_{2} \mathrm{O}$ or $42 \%$ improvement of PImax in the intervention group compared with $9 \mathrm{~cm} \mathrm{H}_{2} \mathrm{O}$ or
$17 \%$ in the control group. In a recent meta-analysis, the improvements were $18 \mathrm{~cm} \mathrm{H}_{2} \mathrm{O}$ (29\%) in the intervention group and only $5 \mathrm{~cm} \mathrm{H}_{2} \mathrm{O}(8 \%)$ in the control group. ${ }^{9}$ This resulted in PImax effect sizes of 1.07 in the current study compared with 0.73 in the

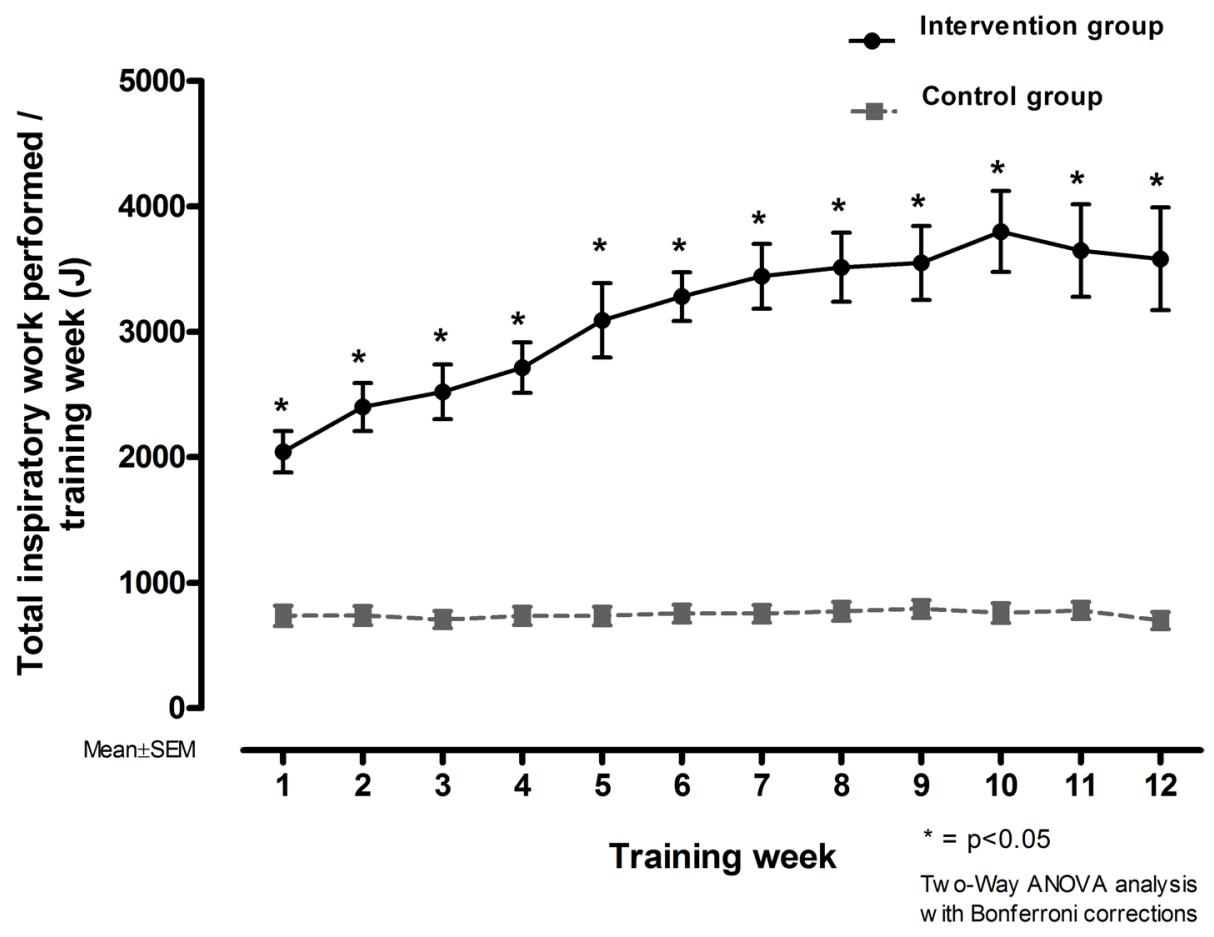

Figure 2 Progression of total work performed during the inspiratory muscle training programme in the intervention and control group. * $\mathrm{P}<0.05$ between groups based on a two-way analysis of variance (ANOVA) analysis with Bonferroni corrections of post-hoc tests, values represented as mean \pm SEM. 
Table 3 Changes in respiratory muscle function

\begin{tabular}{|c|c|c|c|c|c|c|}
\hline \multirow[b]{2}{*}{ Variables } & \multicolumn{2}{|c|}{ Intervention group } & \multicolumn{2}{|c|}{ Control group } & \multirow{2}{*}{$\begin{array}{l}\text { Adjusted difference } \\
(95 \% \mathrm{Cl}) \text { at post training }\end{array}$} & \multirow[b]{2}{*}{$P$ values* } \\
\hline & Pre training & Post training & Pre training & Post training & & \\
\hline $\operatorname{PImax}\left(\mathrm{cm} \mathrm{H}_{2} \mathrm{O}\right)$ & $53(14)$ & $75(19) \dagger$ & $52(11)$ & $61(13) \dagger$ & 14 (10 to 18$)$ & $<0.001$ \\
\hline PEmax $\left(\mathrm{cm} \mathrm{H}_{2} \mathrm{O}\right)$ & $110(40)$ & $127(52) \dagger$ & $104(30)$ & $117(35) \dagger$ & $3(-7$ to 14$)$ & 0.531 \\
\hline Endurance breathing (s) & $240(108)$ & $593(270) \dagger$ & $251(96)$ & $413(236) \dagger$ & 189 (114 to 265$)$ & $<0.001$ \\
\hline Ti/Ttot (\%) & $31(17)$ & $21(14) \dagger$ & $34(19)$ & $28(17) \dagger$ & $-5.6(-9.3$ to -1.9$)$ & 0.003 \\
\hline Total work (J) & $78(83)$ & $258(153) \dagger$ & $79(97)$ & $159(162) \dagger$ & 100 (57 to 142$)$ & $<0.001$ \\
\hline Average power (W) & $1.8(1.4)$ & $3.1(1.7) \dagger$ & $1.5(1.5)$ & $2.1(1.6) \dagger$ & 0.8 (0.4 to 1.1$)$ & $<0.001$ \\
\hline
\end{tabular}

Data are presented as mean (SD) and mean $(95 \% \mathrm{Cl})$.Analyses are based on 174 (PImax, 100\% of completers) patients.

${ }^{*} P$ values are reported for between-group comparisons (analysis of covariance of post-training values adjusted for baseline values as covariates).

tA statistically significant within-group difference $(p<0.05)$.

PEmax, maximal expiratory mouth pressure; PImax , maximal inspiratory mouth pressure; Ti/Ttot, inspiratory duty cycle.

meta-analysis. The larger improvements in the control group $(17 \%$ in our study vs $8 \%$ in the meta-analysis) might be explained by the fact that not all control groups in the meta-analysis performed a sham training intervention. Moreover in the current study, all patients (intervention and control group) were maximally familiarised with the PImax measurements which were performed every week during the intervention period. The larger improvements in our intervention group in comparison to previous studies (42\% vs $29 \%$ ) might be related to the well-controlled nature of the IMT programme (despite the fact that it was not performed fully supervised). ${ }^{18}$ The magnitude of improvements in PImax is comparable to previous studies involving this IMT protocol in patients with COPD. ${ }^{18}{ }^{30}$ The large effect size in endurance breathing time of 0.79 was somewhat smaller compared with the results of the meta-analysis (effect size: 1.05). ${ }^{9}$ This might again be partly explained by the fact that all patients in our study (in contrast to the studies included in the meta-analysis) participated in a sham control training. This sham intervention (ie, performing full vital capacity inspirations while generating high inspiratory flow rates) might even have constituted an endurance-type training stimulus for these patients in addition to the endurance-type training stimulus provided by the GET sessions itself.

Beyond additional improvements in exercise capacity and symptoms during the endurance cycling test and respiratory muscle function, we did not observe any further improvements in quality-of-life domains (including dyspnoea subscales). It might be that the substantial improvements in quality-of-life scores in the control group in response to the comprehensive rehabilitation intervention were already approaching the ceiling effect for improvement. In analogy with the absence of additional improvements in the
6MWD, this might be related to a limited sensitivity of the applied questionnaire (CRQ) to pick up additional improvements on top of an already very significant and clinically important effect. This might be improved in future studies by using other potentially more sensitive dyspnoea scales. One option would be to use scales that are specifically designed to detect different gradations of change like the transitional dyspnoea index. ${ }^{31}$ Another excellent emerging option is the multidimensional dyspnoea profile which can be specifically focused to assess symptoms during predefined periods (eg, at standardised times during an exercise test). ${ }^{32}$

With regard to improvements in daily physical activity, we observed similar improvements in both groups. We know that physical activity is a complex behaviour that is determined by multiple factors. A systematic review demonstrated that large improvements in exercise capacity and symptoms after rehabilitation interventions only result in relatively modest increases in physical activity compared with control groups in patients with COPD. ${ }^{33}$ We therefore believe that it would be unrealistic to expect that the relatively small additional improvements in endurance exercise capacity and dyspnoea symptoms after adjunctive IMT (compared with effects of PR vs control groups) would easily translate into greater participation in daily physical activity.

\section{Correlates of improvements in respiratory muscle function and impact of 'centre' on outcomes}

A significant relationship was observed between the improvements in PImax and both training quality during IMT (ie, total inspiratory work performed/session, average peak power/ session) as well as progression of training intensity. Our results
A

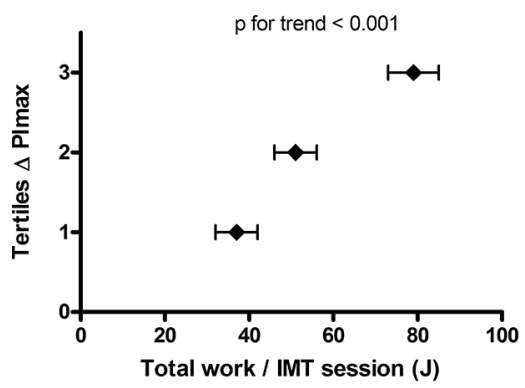

B

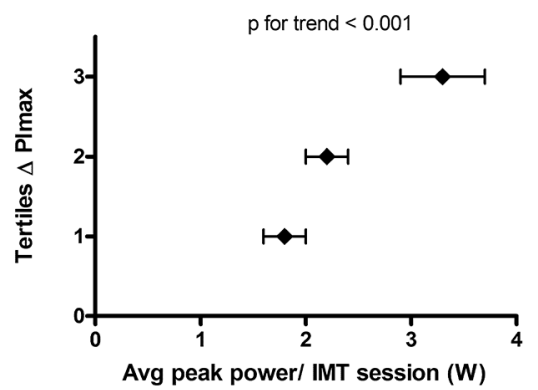

C

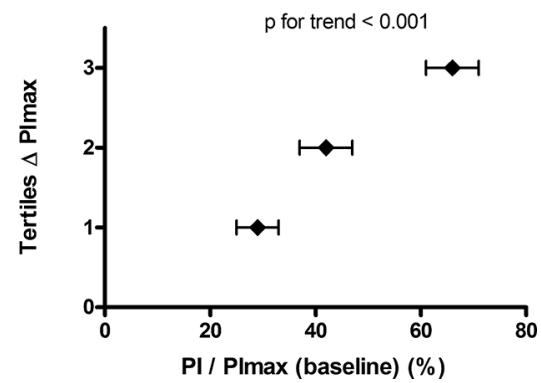

Figure 3 Relationships of training quality indices (total work performed per inspiratory muscle training (IMT) session, average peak power per IMT session; (A, B), and training intensity (defined as inspiratory load (PI) during the final IMT sessions expressed as \% of baseline PImax; $\mathrm{C}$ ), with improvements in PImax. Tertiles based on improvements in PImax were defined as follows: 1 st tertile: $\Delta$ PImax $\leq 7 \mathrm{~cm} \mathrm{H} \mathrm{H}_{2} \mathrm{O}(\mathrm{n}=56 ; 29 \%$ intervention group and $71 \%$ control group), 2nd tertile $\triangle \mathrm{PImax} 8-20 \mathrm{~cm} \mathrm{H}_{2} \mathrm{O}(\mathrm{n}=60 ; 48 \%$ intervention group and $52 \%$ control group), 3rd tertile: $\Delta \mathrm{PImax} \geq 21$ $\mathrm{Cm} \mathrm{H}_{2} \mathrm{O}(\mathrm{n}=55 ; 78 \%$ intervention group and $22 \%$ control group). Data are presented as mean $\pm \mathrm{SEM}$. 

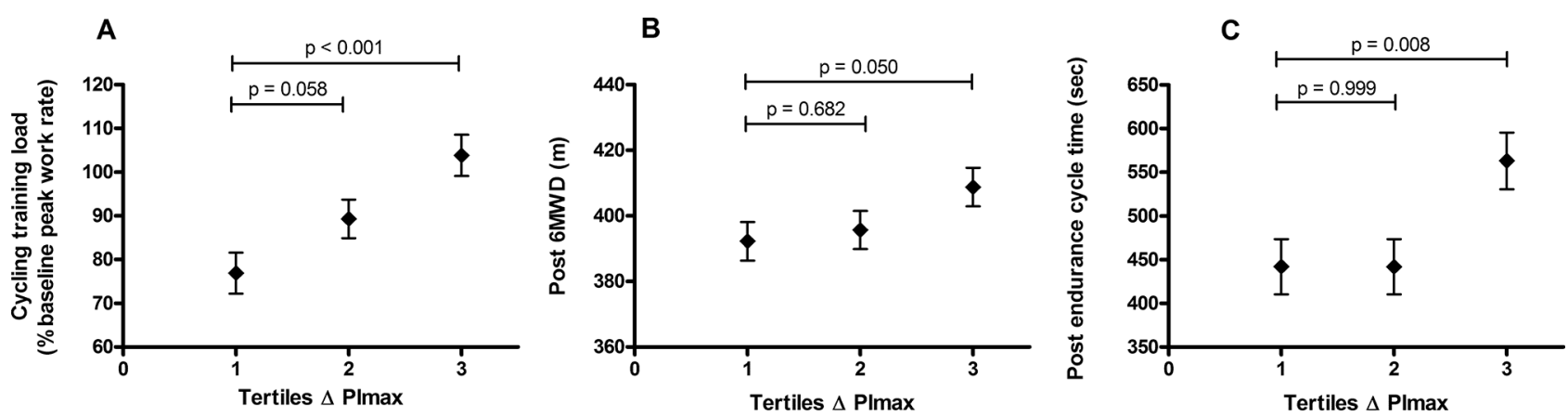

Figure 4 Relationships between improvements in PImax and cycling training load during the last week of general exercise training (A), 6 min walking distance (6MWD) (B) and endurance cycling time after training (C). Tertiles based on improvements in PImax were defined as follows: 1st tertile: $\triangle$ PImax $\leq 7 \mathrm{~cm} \mathrm{H}_{2} \mathrm{O}$ ( $\mathrm{n}=56 ; 29 \%$ intervention group and $71 \%$ control group), 2nd tertile $\triangle$ PImax $8-20 \mathrm{~cm} \mathrm{H}_{2} \mathrm{O}$ ( $\mathrm{n}=60 ; 48 \%$ intervention group and $52 \%$ control group), 3rd tertile: $\triangle \mathrm{PImax} \geq 21 \mathrm{~cm} \mathrm{H}_{2} \mathrm{O}(\mathrm{n}=55 ; 78 \%$ intervention group and $22 \%$ control group). Data are presented as mean \pm SEM.

emphasise that external work and power are important training quality parameters of the training stimulus that is delivered to the inspiratory muscles. Training volume or compliance (number of completed training sessions) was not significantly related with the magnitude of changes in PImax. Interestingly, we found in a multiple regression model that from all training variables the total work performed (reflecting pressure and volume responses during individual sessions in combination with the number of training sessions) was the strongest predictor of improvements in PImax (see online supplementary table E5). This underlines that monitoring the quality of the training (total work performed) during IMT is of utmost importance to ensure meaningful training adaptations in response to the intervention.

One participating centre that contributed to $36 \%$ of all inclusions offered a 20 -session GET programme while all other centres offered a 36-session programme. A priori, we had hypothesised that adjunctive effects in the centre offering fewer training sessions would be smaller. We concluded this since we expected that it would take some weeks before the reductions in exertional dyspnoea induced by IMT would result in higher training intensities during bicycle and treadmill endurance exercises that to be tolerated by the participants of the PR programme. Unexpectedly, compared with the centre that offered 20 sessions, those offering more sessions achieved both consistently larger and consistently smaller between-group differences in several relevant outcomes (ie, increases in PImax, bicycle exercise intensity during PR sessions, 6MWD and endurance cycling time; see online supplementary table E4) compared with the centre that offered 20 sessions. The uniformity of these data (ie, differences in these variables were either consistently larger or smaller) is in line with the presented relationships between improvements achieved in PImax, on the one hand, and the ability to increase exercise intensity during GET and resulting improvements in exercise capacity, on the other hand (figure 4). From these observations, we conclude that factors other than the number of sessions offered (such as contrasts in symptom-based training intensity during GET sessions between groups) probably contributed to the interaction between centre and training outcomes.

\section{Strengths and limitations}

One of the major advantages of the IMT intervention compared with previous trials was the ability to record and store training parameters with the training device. The information of recorded data from IMT sessions performed in the home setting was used to provide patients with feedback on their performance during supervised sessions. It also allowed us to reduce the total volume of supervised sessions to only $7 \%$ of all training sessions, thereby reducing burden on healthcare providers offering the intervention. Both groups had a high and similar IMT training compliance (training volume). Good compliance in the control group was probably partly related to the fact that all patients were led to believe that they were participating in an active intervention. Fifty-five per cent of patients in the intervention group met the target training intensity (inspiratory load of $\geq 50 \%$ PImax) already from the second week of training and increased their training intensity to an average of $84 \%$ of their baseline PImax by the end of the IMT intervention (see online supplementary figure E2). Moreover, patients in the intervention group performed the IMT sessions at this high training intensity while reporting only moderate $(3.5 \pm 0.3)$ perceived inspiratory effort scores. The relatively low effort scores at high training intensities might be related to the loading characteristics of the device, providing an adequate volume reward with every inspiratory effort. These factors taken together probably contributed to the good compliance with the training protocol in the intervention group and are in line with a previous study using a similar training protocol. ${ }^{18}$ The proportion of patients who discontinued the intervention due to lack of motivation was similarly small in both groups (4\% and 5\% in intervention and control group, respectively). Collectively these observations support the clinical feasibility of implementing this controlled, high-intensity adjunctive IMT protocol during a rehabilitation programme in these patients.

Another strength of the study was that the physiotherapists or exercise specialists who supervised the GET were blinded to group allocation. Training intensity during GET was gradually increased during the course of the programme based on highest tolerable dyspnea symptoms assessed with Borg CR-10 symptom scales. ${ }^{34}$ The fact that we observed slightly higher (though not significantly different) training intensities especially on the bicycle in the intervention group is another indirect indication that participation in the IMT intervention probably impacted on activity-related symptoms. Another strength of the study is that patient selection, study design, training methods and selected outcomes in the present study followed recent recommendations for this type of intervention. ${ }^{791524}$ A limitation of this approach is that the results are only applicable to a selective group of patients with COPD who have inspiratory muscle weakness. This is a minority of patients since only $22 \%$ of all patients with COPD met the criteria to participate in the study. This limits the external validity of our findings to this specific subgroup of patients. 
This was also the main reason that the recruitment period had to be extended from 2 to 4 years. Given the absence of any contrast between groups on the primary outcome, we do not believe that the study was underpowered for the primary outcome and that a larger sample might have been necessary to detect an effect on 6MWD. We rather believe that different outcomes (ie, endurance cycling or walking test) should be chosen as a primary outcome for future trials of adjunctive interventions during PR. ${ }^{28}$ We do also not believe that a non-inferiority trial would be valuable in this specific setting given the initial hypothesis of the study. Our main research question was whether adjunctive treatments can enhance the effectiveness of PR in selected patients for functional outcomes. The effectiveness of our 'control intervention' (PR) on 6MWD and quality of life has already repeatedly been demonstrated. ${ }^{8}$

Clinical relevance for an individual patient will eventually depend on how the achieved benefits are valued compared with both the efforts that had to be invested and the potential discomfort associated with the intervention. Another limitation of the study is that satisfaction of patients with the adjunctive intervention was not directly assessed. Finally, we did not perform serial IC measurements during exercise. This would have made it possible to evaluate whether (1) these severely hyperinflated patients did dynamically hyperinflate during exercise breathing and whether this might be a patient characteristic influencing the intervention effect and (2) whether the combined intervention was able to modify breathing pattern and the degree of dynamic hyperinflation during exercise hyperpnoea. These measurements could be performed in future studies and might facilitate identification of patients who are more or less likely to respond to the adjunct intervention.

\section{Clinical implications and general conclusion}

The results of this study do not support the addition of IMT to PR to further enhance the 6MWD. At the same time we believe that the improvements in our secondary outcome (constant workrate endurance cycling) were not negligible (50\% greater than rehabilitation alone). We believe that this is an important finding that could guide researchers towards identifying more appropriate primary outcomes for future studies of adjunctive interventions to PR. The multidimensional dyspnoea profile ${ }^{32}$ might be be an interesting option to assess differences in symptoms at standardised times during an exercise test. The overall good training compliance indicates that it was feasible to implement this adjunctive IMT intervention on a relatively large scale. Across the different centres it was furthermore feasible to implement simple and sensitive clinical assessments of respiratory muscle function (both strength and endurance). The study also highlights the benefits of closely monitoring and controlling IMT sessions since both quality and intensity of training sessions were strongly associated with improvements in respiratory muscle function. Additionally, improvements in PImax were related to the progression in cycling training intensity during the GET programme, as well as to increases in 6MWD and endurance cycling time after the training period.

We also believe that (in addition to using endurance exercise tests as primary outcomes) patient satisfaction with adjunctive interventions should be included as an additional important outcome in similar studies in the future.

\footnotetext{
Author affiliations

${ }^{1}$ Respiratory Rehabilitation and Respiratory Division, University Hospital Leuven, Leuven, Belgium

${ }^{2} \mathrm{KU}$ Leuven - University of Leuven, Department of Rehabilitation Sciences, Faculty of
}

Movement and Rehabilitation Sciences, Leuven, Belgium

${ }^{3}$ Department of Physical Therapy, Faculty of Allied Health Sciences, Thammasat University, Pathumthani, Thailand

${ }^{4}$ Department of Human Sciences and Public Health, Faculty of Health and Social Sciences, Bournemouth University, Bournemouth, UK

${ }^{5}$ Centre de recherche, Institut Universitaire de Cardiologie et de Pneumologie de Québec, Université Laval, Quebec, Canada

${ }^{6}$ Department of Respiratory Medicine, Ghent University Hospital, Ghent, Belgium ${ }^{7}$ Department of Pulmonary Diseases, Radboud University Nijmegen Medical Centre, Nijmegen, The Netherlands

${ }^{8}$ Department of Respiratory Medicine and Pulmonary Rehabilitation, Schoen Klinik Berchtesgadener Land, Schoenau am Koenigssee, Germany

${ }^{9}$ German Center for Lung Research (DZL), Universities of Giessen and Marburg Lung Center (UGMLC), Giessen, Germany

${ }^{10}$ Department of Prevention, Rehabilitation and Sports Medicine, Technical University of Munich (TUM), Munich, Germany

Acknowledgements The authors acknowledge HaB International (Southam, UK) and McRoberts (The Hague, The Netherlands) for providing the equipment for the testing and training in this study. On behalf of IMTCO investigators, the authors would like to acknowledge the physiotherapists from all centres for performing the pulmonary rehabilitation programme, and all measurements of included patients as blinded outcome assessors.

Contributors RG, DL and NC had full access to all of the data in the study and take responsibility for the integrity of the data and the accuracy of the data analysis. DL, NC, RG, MD and AM contributed substantially to the study design. DL, NC, $H D, F M, D S, S V, H v H, L G$, TS and RG provided data collection. DL, RG, NC and HD contributed to the data analysis and interpretation. DL, RG and NC drafted the report, and all authors then critically reviewed it for important intellectual content.

Funding DL and HD are postdoctoral fellows of Research Foundation Flanders. $\mathrm{HaB}$ International (Southam, UK) and McRoberts (The Hague, The Netherlands) provided equipment for testing and training in this study on loan. This study was further supported by local funds throughout the participating centres. The following specific funding sources were reported: University Hospital Leuven, Belgium (FWO grant GOA4516N en KU Leuven grant C22/15/035); Ghent University Hospital, Belgium (UZ Gent grant FS/LGZ/994); Institut Universitaire de Cardiologie et de Pneumologie de Québec, Université Laval, Quebec, Canada (Ordre professionnel de la physiothérapie du Québec).

Disclaimer None of the sponsors had any role in the preparation of the trial design, patient recruitment, data collection, data analysis, interpretation of the data, approval of the report or the decision to submit this manuscript for publication.

Competing interests AM acknowledges a previous (now expired) beneficial interest in the POWERbreathe inspiratory muscle trainers in the form of a share of royalty income to the University of Birmingham, and a potential share of royalty income to Brunel University. In the past, she has also provided consultancy services to POWERbreathe International, but no longer does so. She is named on two patents relating to POWERbreathe products, including the device used in the present study, as well as being the author of two books on inspiratory muscle training. FM reports research support from Boehringer Ingelheim, GlaxoSmithKline, AstraZeneca, Grifols and Novartis, advisory board participation for Boehringer Ingelheim and GlaxoSmithKline, and speaking engagements for Boehringer Ingelheim, GlaxoSmithKline, AstraZeneca, Grifols and Novartis.

\section{Patient consent Obtained.}

Ethics approval University Hospital Leuven Institutional Review Board (approval number ML7489).

Provenance and peer review Not commissioned; externally peer reviewed.

(c) Article author(s) (or their employer(s) unless otherwise stated in the text of the article) 2018. All rights reserved. No commercial use is permitted unless otherwise expressly granted.

\section{REFERENCES}

1 Vogelmeier CF, Criner GJ, Martinez FJ, et al. Global strategy for the diagnosis, management, and prevention of chronic obstructive lung disease 2017 report: GOLD executive summary. Am J Respir Crit Care Med 2017.

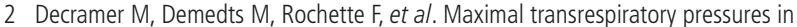
obstructive lung disease. Bull Eur Physiopathol Respir 1980;16:479-90.

3 Hamilton AL, Killian KJ, Summers E, et al. Muscle strength, symptom intensity, and exercise capacity in patients with cardiorespiratory disorders. Am J Respir Crit Care Med 1995;152:2021-31.

4 Killian KJ, Jones NL. Respiratory muscles and dyspnea. Clin Chest Med 1988:9:237-48.

5 Gosselink R, Troosters T, Decramer M. Peripheral muscle weakness contributes to exercise limitation in COPD. Am J Respir Crit Care Med 1996;153:976-80. 
6 Laghi F, Tobin MJ. Disorders of the respiratory muscles. Am J Respir Crit Care Med 2003;168:10-48.

7 Spruit MA, Singh SJ, Garvey C, et al. An official American Thoracic Society/European Respiratory Society statement: key concepts and advances in pulmonary rehabilitation. Am J Respir Crit Care Med 2013;188:e13-e64.

8 McCarthy B, Casey D, Devane D, et al. Pulmonary rehabilitation for chronic obstructive pulmonary disease. Cochrane Database Syst Rev 2015:CD003793. CD003793.

9 Gosselink R, De Vos J, van den Heuvel SP, et al. Impact of inspiratory muscle training in patients with COPD: what is the evidence? Eur Respir J 2011;37:416-25.

10 Ambrosino N. The case for inspiratory muscle training in COPD. Eur Respir J 2011:37:233-5.

11 McConnell AK. CrossTalk opposing view: respiratory muscle training does improve exercise tolerance. J Physiol 2012;590:3397-8.

12 Patel MS, Hart N, Polkey MI. CrossTalk proposal: training the respiratory muscles does not improve exercise tolerance. J Physiol 2012;590:3393-5.

13 Polkey MI, Moxham J, Green M. The case against inspiratory muscle training in COPD. Against. Eur Respir J 2011;37:236-7.

14 Spruit MA, Pitta F, Garvey C, et al. American Association of C, Pulmonary R, Assembly ATSPR and the ERSCAt. Differences in content and organisational aspects of pulmonary rehabilitation programmes. Eur Respir J 2014;43:1326-37.

15 Decramer M. Response of the respiratory muscles to rehabilitation in COPD. J Appl Physiol 2009;107:971-6.

16 Charususin N, Gosselink R, Decramer M, et al. Inspiratory muscle training protocol for patients with chronic obstructive pulmonary disease (IMTCO study): a multicentre randomised controlled trial. BMJ Open 2013;3:e003101.

17 Fregni $\mathrm{F}$, Imamura $\mathrm{M}$, Chien $\mathrm{HF}$, et al. Challenges and recommendations for placebo controls in randomized trials in physical and rehabilitation medicine: a report of the international placebo symposium working group. Am J Phys Med Rehabil 2010;89:160-72.

18 Langer D, Charususin N, Jácome C, et al. Efficacy of a Novel Method for Inspiratory Muscle Training in People With Chronic Obstructive Pulmonary Disease. Phys Ther 2015:95:1264-73.

19 Troosters T, Gosselink R, Decramer M. Six minute walking distance in healthy elderly subjects. Eur Respir J 1999;14:270-4.

20 Puhan MA, Chandra D, Mosenifar Z, et al. The minimal important difference of exercise tests in severe COPD. Eur Respir J 2011;37:784-90.
21 Alshurafa M, Briel M, Akl EA, et al. Inconsistent definitions for intention-to-treat in relation to missing outcome data: systematic review of the methods literature. PLOS One 2012;7:e49163.

22 Vickers AJ, Altman DG. Statistics notes: Analysing controlled trials with baseline and follow up measurements. BMJ 2001;323:1123-4.

23 Holland AE, Nici L. The return of the minimum clinically important difference for 6-minute-walk distance in chronic obstructive pulmonary disease. Am J Respir Crit Care Med 2013;187:335-6.

24 Camillo CA, Osadnik CR, van Remoortel H, et al. Effect of "add-on" interventions on exercise training in individuals with COPD: a systematic review. ERJ Open Res 2016:2:00078-2015.

25 Cazzola M, MacNee W, Martinez FJ, et al. Outcomes for COPD pharmacological trials: from lung function to biomarkers. Eur Respir J 2008:31:416-69.

26 Laviolette L, Bourbeau J, Bernard S, et al. Assessing the impact of pulmonary rehabilitation on functional status in COPD. Thorax 2008;63:115-21.

27 Pepin V, Brodeur J, Lacasse Y, et al. Six-minute walking versus shuttle walking: responsiveness to bronchodilation in chronic obstructive pulmonary disease. Thorax 2007:62:291-8.

28 Maltais F, Hamilton A, Trampisch M, et al. Effect of Once-Daily Tiotropium and Olodaterol, Alone and Combined With Exercise Training, on Two Measures of Walking Capacity in Patients With COPD. Chest 2016;150:853A.

29 Holland AE, Spruit MA, Troosters T, et al. An official European Respiratory Society/ American Thoracic Society technical standard: field walking tests in chronic respiratory disease. Eur Respir J 2014;44:1428-46.

30 Charususin N, Gosselink R, McConnell A, et al. Inspiratory muscle training improves breathing pattern during exercise in COPD patients. Eur Respir J 2016;47:1261-4.

31 Mahler DA, Witek TJ. The MCID of the transition dyspnea index is a total score of one unit. COPD 2005;2:99-103.

32 Banzett RB, O'Donnell CR, Guilfoyle TE, et al. Multidimensional Dyspnea Profile: an instrument for clinical and laboratory research. Eur Respir J 2015;45:1681-91.

33 Cindy Ng LW, Mackney J, Jenkins S, et al. Does exercise training change physical activity in people with COPD? A systematic review and meta-analysis. Chron Respir Dis 2012;9:17-26.

34 Borg GA. Psychophysical bases of perceived exertion. Med Sci Sports Exerc 1982;14:377-81. 